\section{Watching Them Wash: Description of a Hand Hygiene Observation Program}

To the Editor-Optimizing compliance with hand hygiene is the most critical aspect of any infection control program., ${ }^{1,2}$ An essential but difficult element of an effective hospital hand hygiene program is assessing compliance with hand hygiene. Currently, there is no generally accepted standardized method to assess compliance with hand hygiene in hospitals, and multiple approaches have been tried, including direct observation, self-reporting by healthcare workers, and surrogate measurements, such as electronic counting devices on soap dispensers or hand hygiene product use. ${ }^{1,3}$ The gold standard is direct observation, although this method is associated with high resource and labor costs. ${ }^{1,3}$ We describe our experience with a hand hygiene program based on direct observation at an 820-bed, urban tertiary care center in central Virginia.

Our program consists of a team of roving observers, typically college and graduate students, who are paid an hourly wage. Daily assignments are made; the assignment may be a randomly chosen hospital unit or a unit specifically chosen because of a recent increase in infection or recent findings of poor hand hygiene compliance. Each observer was trained by the same nurse epidemiologist to minimize interobserver variability, and the unit of observation was compliance with hand hygiene on room entry and exit. We reviewed data from a recent 1-year period (October 2007 through September 2008) gathered in our hand hygiene observation program, including information on overall compliance rates for healthcare providers stratified by specialty site and job classification. We also reviewed data on overall costs of the program for the same period.

During the study period, a total of 32,400 individual hand hygiene observations were made. The overall compliance was $84 \%$, with respiratory therapists having the highest overall compliance $(87 \%)$ and physicians having the lowest overall compliance $(66 \%)$. The total compliance by healthcare job for the period of interest is noted in the Table.

In terms of compliance per unit, the worst area of compliance was the emergency department (53\%), with the highest compliance on that unit by nurses $(60 \%)$ and the lowest compliance by radiology technicians (26\%). Our bone marrow transplant unit had the highest overall compliance with a rate of $93 \%$, with physicians on that unit demonstrating $100 \%$ compliance.

The program employed 12 staff observers (typically 3 at any given time) who worked a total of 2,074 hours for a combined cost of $\$ 21,252$. This amounts to $\$ 0.66$ per observation.
Although direct observation has the advantages of being able to determine exactly who is complying with hand hygiene and of permitting for the assessment of technique, it is associated with relatively high resource and labor costs. In addition, this form of observation is potentially limited by the Hawthorne effect (wherein individuals who are being observed change their normal behavior, thereby falsely elevating compliance rates) and potentially limited by interobserver variability. ${ }^{3}$ Also, it is more difficult to observe healthcare workers in a ward setting where visibility into the room is typically obstructed. We attempted to control for interobserver variability by having all observers trained by a single nurse epidemiologist. In addition, by using dedicated compliance monitors who were student workers, we were able to accrue a large number of observations while keeping costs low $(32,400$ individual hand hygiene observations over the course of this 1-year study at a cost of $\$ 0.66$ per observation). At our institution, these data are directly fed back to healthcare workers via weekly e-mail communication with physicians and senior staff members, nursing unit-specific scorecards, and intensive care unit-specific infection control posters.

Our hand hygiene observation program, like those of many other hospitals, is based on direct observation, but it differs in its approach by using students specifically hired for this purpose. This allowed us to perform a large number of observations at a relatively low cost.

\section{ACKNOWLEDGMENTS}

Potential conflicts of interest. All authors report no conflicts of interest relevant to this article.

Michael P. Stevens, MD; John D. Hunter; Janis F. Ober, RN; Gonzalo Bearman, MD, MPH; Michael B. Edmond, MD, MPH, MPA

From the Division of Infectious Diseases, Virginia Commonwealth University Medical Center (M.P.S., J.F.O., G.B., M.B.E.), and Virginia Commonwealth University (J.D.H.), Richmond, Virginia.

Address reprint requests to Michael P. Stevens, MD, $1201 \mathrm{E}$ Marshall St, PO Box 980019, Richmond, VA 23298-0019 (mstevens2@mcvh-vcu.edu).

Presented in part: 19th Annual Scientific Meeting of the Society for Healthcare Epidemiology; San Diego, California; March 19-22, 2009. Infect Control Hosp Epidemiol 2010; 31:198-199

(C) 2009 by The Society for Healthcare Epidemiology of America. All rights reserved. 0899-823X/2010/3102-0018\$15.00. DOI: $10.1086 / 650378$

\section{REFERENCES}

1. Consensus Measurement in Hand Hygiene Project Expert Advisory Panel. Measuring hand hygiene adherence: overcoming the challenges. The Joint Commission. 2009. Available at: http://www.jointcommission.org/ 
PatientSafety/InfectionControl/hh_monograph.htm. Accessed August 3, 2009.

2. Pittet D. Compliance with hand disinfection and its impact on hospitalacquired infections. J Hosp Infect 2001;48(Suppl A):S40-S46.

3. Haas JP, Larson EL. Measurement of compliance with hand hygiene. $J$ Hosp Infect 2007;66:6-14.

\section{Infection Prevention Education: Are We Neglecting It?}

To the Editor-Healthcare-associated infections pose a formidable challenge to the healthcare industry, with a significant impact on patient outcomes. ${ }^{1}$ Preventive measures such as hand hygiene are of paramount importance in reducing healthcare-associated infections, but despite the utility of this simple procedure, compliance rates are suboptimal at many healthcare institutions. ${ }^{2}$ Part of the reason for nonadherence to basic measures of infection prevention is lack of knowledge among physicians. ${ }^{3}$ Infection prevention education has the potential to be effective, but didactic teaching sessions specifically intended for physicians in training are limited. ${ }^{4} \mathrm{Few}$ studies have assessed what physicians in training know about infection prevention, and the existing evidence reveals that physicians lack sufficient knowledge about this topic. ${ }^{5-8}$ Moreover, the data in the medical literature tend to be more reflective of norms within the United Kingdom and other European countries than of norms identified within the United States. ${ }^{4-8}$ Notably, there are no specific educational guidelines set by the Accreditation Council for Graduate Medical Education, which cites a need for residents to "demonstrate knowledge of established and evolving biomedical, clinical, epidemiological, and social-behavioral sciences." This broad objective would encompass education about infection prevention. However, we are not aware of any structured curricula that residency programs in the United States have adopted to educate physicians about infection prevention. We therefore conducted a cross-sectional study to gather information about how internal medicine residency programs in the United States educate house staff on these principles.

There are 381 internal medicine residency training programs in the United States and its territories. Essential contact information with regard to the programs was obtained from the Fellowship and Residency Electronic Interactive Database. ${ }^{10}$ This was done after approval from our institutional review board. US internal medicine residency program directors were sent a cover letter with a survey and a preaddressed postcard. The program directors completed the form and returned it to us. In an effort to ensure anonymity, instructions were provided to mail the postcard separately.

The letter conveyed the intent of gathering information about the different ways residency programs educated house
TABLE. Compliance with Hand Hygiene, by Healthcare Job

\begin{tabular}{lcc}
\hline Job title & $\begin{array}{c}\text { No. of } \\
\text { observations }\end{array}$ & Compliance, \% \\
\hline Nurse & 25,234 & 87.4 \\
Physician & 4,511 & 66.3 \\
Radiology technician & 314 & 67.8 \\
Respiratory therapist & 829 & 87.5 \\
Physical therapist & 245 & 86.5 \\
Other & 1,267 & 81.7 \\
Total & 32,400 & 84.0 \\
\hline
\end{tabular}

staff about basic principles of infection prevention. Specifically, the letter stated that, for the purposes of this survey:

[T] he term 'Infection Prevention' generally encompasses (but is not strictly limited to) the following basic principles:

Hand Hygiene: Use of antibacterial soap and water or hand sanitizer before and after patient/environment contact,

Precautions: Types of precautions (ie, contact airborne, droplet isolation, etc), and

Multi-Drug Resistant Organisms: Understanding the significance of drug resistant organisms such as MRSA and VRE.

Commonly conceivable means of educating house staff were listed on the questionnaire (Table 1). The last option, "Other (please specify)," was kept open-ended to gather additional means of education on infection prevention. The survey was prepared by the investigators and reviewed by a variety of teaching physicians and a public health professional prior to distribution.

From the 381 internal medicine residency training programs (and their respective program directors) in the United States and its territories, we obtained responses to the survey from 158 program directors (41.4\%) within a 3-month period. No responses were obtained thereafter. Responses to the questionnaire survey have been tabulated (Table 1). Ad hoc responses within the "Other (please specify)" category were grouped on the basis of similar themes (not verbatim), including the following commonly conceivable means of educating house staff:

1. Activities occurring during clinical rotations (rounds on floor, morning report, intensive care rotation): 9 independent responses.

2. Incorporated into the agenda during resident meetings/conferences (meetings with faculty, non-core lectures, "continuous" reinforcement--not otherwise specified): 6 independent responses.

3. Captured by general hospital safety campaigns (Quality Improvement, Quality Assurance, preparation for The Joint Commission inspection): 6 independent responses.

4. Hand washing monitoring on floors providing direct feedback: 1 independent response.

There is increasing awareness of the importance of infection prevention in healthcare facilities. Although many states 